\title{
Dipolar antiferromagnetism and quantum criticality in $\mathrm{LiErF}_{4}$
}

\author{
Conradin Kraemer, ${ }^{1,2}$ Neda Nikseresht, ${ }^{1}$ Julian O. Piatek ${ }^{1}$ \\ Nikolay Tsyrulin, ${ }^{1}$ Bastien Dalla Piazza, ${ }^{1}$ Klaus Kiefer, ${ }^{3}$ \\ Bastian Klemke, ${ }^{3}$ Thomas F. Rosenbaum, ${ }^{4}$ Gabriel Aeppli, ${ }^{5}$ \\ Ché Gannarelli, ${ }^{5}$ Karel Prokes, ${ }^{3}$ Andrey Podlesnyak, ${ }^{6}$ \\ Thierry Strässle, ${ }^{2}$ Lukas Keller, ${ }^{2}$ Oksana Zaharko, ${ }^{2}$ \\ Karl W. Krämer, ${ }^{7}$ Henrik M. Rønnow, ${ }^{1 *}$ \\ ${ }^{1}$ Laboratory for Quantum Magnetism, Ecole Polytechnique Fédérale de Lausanne (EPFL), \\ Lausanne, Switzerland \\ ${ }^{2}$ Laboratory for Neutron Scattering, Paul Scherrer Institute, \\ 5232 Villigen, Switzerland \\ ${ }^{3}$ Helmholtz-Zentrum Berlin, 14109 Berlin Wannsee, Germany \\ ${ }^{4}$ James Franck Institute and Department of Physics, University of Chicago, \\ Chicago, Illinois 60637, USA \\ ${ }^{5}$ London Centre for Nanotechnology and Department of Physics and Astronomy, \\ UCL, London, WC1E 6BT, United Kingdom \\ ${ }^{6}$ Oak Ridge National Laboratory, Spallation Neutron Source, Oak Ridge 37831, USA \\ ${ }^{7}$ Department of Chemistry and Biochemistry, University of Bern, 3000 Bern 9, Switzerland
}

*To whom correspondence should be addressed; E-mail: henrik.ronnow@epfl.ch

Magnetism has been predicted to occur in systems where dipolar interactions

dominate exchange. We present neutron scattering, specific heat and magnetic susceptibility data for $\mathrm{LiErF}_{4}$, establishing it as a model dipolar-coupled antiferromagnet with planar spin-anisotropy and a quantum phase transition 
in applied field $H_{c||}=4.0 \pm 0.1$ kOe. We discover non-mean-field critical scaling for the classical phase transition at the antiferromagnetic transition temperature consistent with the $2 \mathrm{D} \mathrm{XY} / h_{4}$ universality class, in accord with this, the quantum phase transition at $\mathbf{H}_{c}$ exhibits three-dimensional classical behaviour. The effective dimensional reduction may be a consequence of the intrinsic frustrated nature of the dipolar interaction, which strengthens the role of fluctuations.

The dipolar force between magnetic moments - a consequence of Maxwell's fundamental laws for electromagnetism - is present in all magnetic systems, from classical to quantum magnets, from bulk materials to nano-particles. Over half a century ago, Luttinger and Tisza (1) discussed whether a polarized state of matter can be induced by classical dipole-dipole interactions alone, and in the absence of short range forces such as exchange interactions. They conjectured that both ferromagnetic (FM) and antiferromagnetic (AFM) order can arise, depending on the geometrical arrangement of the dipoles. When the modern theory of critical phenomena was developed, dipolar-coupled ferromagnets, where the dipoles are atomic magnetic moments, presented material realizations where concepts could be tested. Being three-dimensional systems they were at the upper marginal dimension for the applicability of mean-field (MF) theory. This resulted in logarithmic corrections, which could be calculated exactly and agreed with the measured behaviour around the classical phase transition (2). In the context of quantum phase transitions (QPT), anisotropic dipolar systems are excellent realizations of e.g. the Ising model in a transverse field. In dipolar systems, the anisotropy ratio for the dipolar interaction scales as the square of the anisotropy ratio for response to an external magnetic field, and as a consequence even for modest single-ion anisotropy the dipolar interaction along the hard axis is much smaller than along the easy axis. This hierarchy of scales is much harder to achieve in exchange-coupled systems where the moment-carrying electron wave-functions are responsible 
for both the exchange and single-ion anisotropies.

An excellent testing ground for the physics of dipolar-coupled systems are the lithium rare earth $(R E)$ tetrafluorides, $\mathrm{Li}_{R E \mathrm{~F}_{4}}$, where tightly bound $4 f$ electrons are far enough apart for the dipolar interactions to dominate exchange interactions. Another major advantage of the $\mathrm{Li} R E \mathrm{~F}_{4}$ family is the possibility of isostructural dilution with non-magnetic Yttrium, $\mathrm{Li} R E_{x} \mathrm{Y}_{1-x} \mathrm{~F}_{4}$, permitting experiments from isolated dipoles (3) through disordered interacting dipoles forming spin glass states (4-6), to the undiluted limit $\mathrm{Li} R E \mathrm{~F}_{4}$. To date activity has centered on the Ising-like ferromagnets $\mathrm{LiTbF}_{4}(2)$ and $\mathrm{LiHoF}_{4}(7)$, and their respective dilution series (8). Here we focus on an AFM member of the family $\mathrm{LiErF}_{4}$, and address the magnetic order, the classical phase transition, and the transition and fluctuations about the quantum critical point.

Known as $R E$ :YLF, very dilute $(x<1 \%) \mathrm{Li}_{R} E_{x} \mathrm{Y}_{1-x} \mathrm{~F}_{4}$ is employed commercially in laser technology because of the long lifetimes of the crystal field energy levels of isolated $R E^{3+}$ ions. The crystal field also sets the stage for low-temperature collective properties. The electric field from neighboring ions act differently on the orbital wave-functions, and, restricted by the local symmetry, gives the following crystal field Hamiltonian:

$$
\mathcal{H}_{C F}=\sum_{l=2,4,6} B_{l}^{0} \boldsymbol{O}_{l}^{0}+\sum_{l=4,6} B_{l}^{4}(c) \boldsymbol{O}_{l}^{4}(c)+B_{6}^{4}(s) \boldsymbol{O}_{6}^{4}(s) .
$$

$\boldsymbol{O}_{l}^{m}$ are the Stevens operators with amplitudes $B_{l}^{m}$ (see (9) for definitions). The dominant crystal field component is $\boldsymbol{O}_{2}^{0}=3 J_{z}^{2}-J(J+1)$, where the operator $J_{z}$ is the component of the electronic angular momentum $(J)$ along the $z$ axis. $\mathrm{In} \mathrm{LiHoF}_{4}$, a negative $B_{2}^{0}$ leads to strong $z$-axis Ising anisotropy, whereas a positive $B_{2}^{0}$ leads to planar XY anisotropy in $\mathrm{LiErF}_{4}$.

The full magnetic Hamiltonian for $\mathrm{Li}_{R E \mathrm{~F}_{4}}$ contains crystal field, external field $(H)$ and hyperfine coupling to the nuclear spins, as well as dipolar and exchange interactions: 


$$
\begin{aligned}
\mathcal{H}= & \sum_{i}\left[\mathcal{H}_{C F}\left(\boldsymbol{J}_{i}\right)-g_{L} \mu_{B} \boldsymbol{J}_{i} \cdot H+A \boldsymbol{J}_{i} \cdot \boldsymbol{I}_{i}\right] \\
& -\frac{1}{2} \sum_{i j} \sum_{\alpha \beta} \mathcal{J}_{D} D_{i j}^{\alpha \beta} J_{i \alpha} J_{j \beta}-\frac{1}{2} \sum_{i j, n . n} \mathcal{J}_{\text {ex }} \boldsymbol{J}_{i} \cdot \boldsymbol{J}_{j}
\end{aligned}
$$

where $\boldsymbol{J}_{i}$ and $\boldsymbol{I}_{i}$ are the electronic and nuclear angular momentum operators at site $i$. The electronic dipole moment is given by the angular momentum multiplied by the Bohr magneton $\mu_{B}$ and the Landee factor $g_{L}=1.2$. The strength of hyperfine, exchange and dipolar couplings are defined by $A, \mathcal{J}_{\text {ex }}$ and $\mathcal{J}_{D}$, respectively. The dipole interaction is the tensor:

$$
D_{i j}^{\alpha \beta}=\frac{3\left(r_{i \alpha}-r_{j \alpha}\right)\left(r_{i \beta}-r_{j \beta}\right)-\left|\boldsymbol{r}_{i}-\boldsymbol{r}_{j}\right|^{2} \delta_{\alpha \beta}}{\left|\boldsymbol{r}_{i}-\boldsymbol{r}_{j}\right|^{5}} .
$$

where $\boldsymbol{r}_{i}$ is the position of the $i$ th ion. Its peculiar spatial anisotropy is illustrated in Fig. 1. In $\mathrm{LiHoF}_{4}$, the moments point along $z$, and nearest neighbours (NN) are coupled ferromagnetically. We notice that in $\mathrm{LiErF}_{4}$, where the moments reside in the $a b$-plane, half the NN couplings are AFM, the other half FM, and rotating the moments by 90 degrees switches between FM and AFM interactions. In $\mathrm{LiHoF}_{4}$, the exchange coupling is very small, in the order of $2 \%$ of the effective coupling at zero wave vector (10). Given the similar wavefunctions for Ho and Er, we also expect $\mathcal{J}_{\text {ex }}$ be negligible in $\mathrm{LiErF}_{4}$. The hyperfine coupling $A=0.5(1) \mu \mathrm{eV}$ for ${ }^{167} \operatorname{Er}(11)$ is weaker than $A=3.36 \mu \mathrm{eV}$ in $\mathrm{LiHoF}_{4}(10)$, and, importantly, tunable because crystals can be prepared using ${ }^{168} \mathrm{Er}$ without or ${ }^{167} \mathrm{Er}$ with nuclear moments. Our sample contained natural Er with $23 \%$ nuclear moments.

Limited data exist on the magnetic properties of $\mathrm{LiErF}_{4}$. Electron Paramagnetic Resonance (EPR) (12, 13), susceptibility, ${ }^{7}$ Li Nuclear Magnetic Resonance (NMR) (14) and optical spectroscopy (15) show planar XY anisotropy, but significant variation in the reported anisotropy ratio and the lack of a globally consistent set of crystal field parameters, prevented predictions of low-temperature properties. Susceptibility (16) and specific heat (17) show a transition 
around $380 \mathrm{mK}$. The shape of the specific heat anomaly differs from that of $\mathrm{LiHoF}_{4}$ and 3D exchange-coupled XY materials, but the implied short-range correlations were discussed in terms of unlikely large exchange coupling. Susceptibility suggested AFM order (18), but the magnetic structure has not been determined. To this end, we have undertaken comprehensive neutron scattering, specific heat, and magnetic susceptibility studies.

The crystal field was determined using neutron spectroscopy on a single crystal, providing not only the position of the energy levels, but also the matrix elements of the angular momentum operators, thereby more accurately defining the effective model (Fig. S1 in SOM). The ground state is a Kramers doublet isolated by a $\Delta=2.25 \mathrm{meV}$ gap (Tables S1 and S2 in SOM). Within this sub-space, an effective Hamiltonian for the low-temperature properties can be used in future theoretical work:

$$
\mathcal{H}_{\mathrm{eff}}=\sum_{i j \alpha \beta} \mathcal{J}_{i j}^{\alpha \beta} \sigma_{i}^{\alpha} \sigma_{j}^{\beta}+g_{\perp}\left(\sigma_{i}^{x} B^{x}+\sigma_{i}^{y} B^{y}\right)+g_{\|} \sigma_{i}^{z} B^{z}
$$

where $\sigma_{i}$ denotes the Pauli operators and $\mathcal{J}_{i j}^{\alpha \beta}=\left(\mu_{B} g_{L}\right)^{2} C_{\alpha} C_{\beta} D_{i j}^{\alpha \beta}$ the magnetic coupling tensor between the effective $S=1 / 2$ spins $S^{\alpha}=C_{\alpha} \sigma^{\alpha}$, with parameters calculated from the crystal field refinement: $C_{x}=C_{y}=3.480, C_{z}=0.940, g_{\perp}=2 g_{L} C_{x}=8.35$ and $g_{\|}=$ $2 g_{L} C_{z}=2.25$. We note that albeit the anisotropy ratio of the response to a magnetic field $g_{\perp} / g_{\|}=3.7$ is modest, the anisotropy ratio of the dipolar coupling $\left(g_{\perp} / g_{\|}\right)^{2}=13.8$ becomes large, which is what causes the XY anisotropy.

The magnetic structure was determined by single-crystal and powder neutron diffraction (See Fig. S2 in SOM). Magnetic Bragg peaks at $(h+k+l=$ odd), distinct from the structural peaks $(h+k+l=$ even), prove explicitly AFM order. The single crystal Bragg peak intensities are consistent with the bilayered antiferromagnetic (BLAFM) structure depicted in Fig. 1, also verified by powder diffraction. The BLAFM has 2 equivalent configurations with moments along the $a$-axis or $b$-axis, respectively. A very small field of 300 Oe along the $b$-axis suppresses 
the (100) reflection (Fig. 2E), populating a single $a$-axis domain. Hence, the zero-field structure is a distribution of spatially separated domains with moments along $a$ and $b$, respectively. Full powder refinement yielded an ordered moment $\left\langle J^{x}\right\rangle=2.2 \pm 0.1$, reduced from the value $\left\langle J^{x}\right\rangle_{M F}=3.0$ predicted by a MF calculation.

Fig. 2 shows the phase diagram as function of temperature and fields along the $c$ - and $b$ axes. The transition temperature $T_{N}=373 \pm 5 \mathrm{mK}$ agrees with previous reports. For fields along $c$, the intensity at (010), corresponding to the order parameter squared, disappears in a sharp QPT at $H_{c||}=4.0 \pm 0.1 \mathrm{kOe}$. For fields along $b$, the (100) peak disappears due to monodomain formation. The (003) peak, which is independent of $a b$-domains, decreases towards $H_{c \perp} \simeq 2.1 \mathrm{kOe}$, but a long tail remains to $4 \mathrm{kOe}$. The (103) peak, measuring the uniform FM component, grows towards a kink at $H_{c \perp}$, corresponding to maximal polarization of the ground-state doublet. Above $H_{c \perp}$ a weak linear increase, achieved by mixing-in higher lying crystal field levels, is well reproduced by the MF prediction.

A MF calculation yields the correct BLAFM ordered and a qualitatively correct phase diagram (See SOM). In $\mathrm{LiHoF}_{4}$, a MF treatment accounts for most of the phase diagram except close to $T_{C}$, which is overestimated by $37 \%(10)$. $\mathrm{In} \mathrm{LiErF}_{4}, T_{N}^{\mathrm{mf}}=728 \mathrm{mK}, H_{c \|}^{\mathrm{mf}}=5.25 \mathrm{kOe}$ and $H_{c \perp}^{\mathrm{mf}}=3.25 \mathrm{kOe}$ are all dramatically overestimated. Unlike $\mathrm{LiHoF}_{4}$, any $\mathrm{NN}$ exchange interaction cancels in the BLAFM and cannot fine-tune the phase boundary. Including hyperfine coupling has little effect: $H_{c||}=5.75 \mathrm{kOe}$ and $T_{N}=735 \mathrm{mK}$.

Scaling the temperature and field to match $T_{N}$ and $H_{c}$, the $T_{N}(H)$ curve is well described (Fig. 2A), but the onset of order is more abrupt than the MF prediction (Fig. 2, D and E). Deep in the ordered phase, the unscaled MF calculation works (except for the low-field dip in panel D), which requires further investigation), but around the transition fluctuations gain importance. We measured simultaneously the strength of the critical scattering, whose divergences independently determine $T_{N}$ and $H_{c}$ (Fig. 3, inset), increasing the precision of the extracted critical 
exponents: $\beta_{T}=0.15 \pm 0.02$ for the thermal transition at $H=0 \mathrm{~T}$ and $\beta_{H}=0.31 \pm 0.02$ for the QPT at $H_{c \|}$ (Fig. 3). Both exponents significantly deviate from the MF expectation $\left(\beta^{\mathrm{mf}}=\frac{1}{2}\right)$.

The specific heat shows a pronounced ordering anomaly (Fig. 4), in good accordance with the phase diagram established by neutrons (See Figs. S3 and S4 in SOM). The broad bump around $12 \mathrm{~K}$ is exactly described by our crystal field Hamiltonian. The phonon contribution $\rho T^{3}$ with $\rho=(6.6 \pm 0.1) \cdot 10^{-7} \mathrm{~J} / \mathrm{g} K^{4}$ is much lower than previously reported for $\mathrm{Li} R E \mathrm{~F}_{4}(17)$, where the crystal field contribution was not subtracted. The tail above $T_{N}$ is much more significant than in the FM cases of $\mathrm{LiHoF}_{4}$ and $\mathrm{LiTbF}_{4}(17,19,20)$. Around $T_{N}$, the specific heat follows a universal power law:

$$
C_{p}=A|t|^{-\alpha}+B \quad t=T / T_{c}-1
$$

where $\alpha=-0.28 \pm 0.04$ below and above the transition, while $A_{+} / A_{-}=1.68 \pm 0.04\left(A_{+}\right.$and $A_{-}$are the values of the parameter $A$ above and below the transition temperature, respectively). Subtracting $B$ reveals a crossover to $\alpha=-0.07 \pm 0.05$ for $t>0.03$ (Fig. 4C). Scaling to peak height and temperature, $C_{p}(T)$ curves for different fields collapse to a single unique curve below $T_{N}$ (Fig. 4D).

For the classical phase transition, the exponent $\beta_{T}=0.15 \pm 0.02$ is far from the $\beta=$ $0.3-0.35$ of standard 3D universality classes, and instead falls in the window $\beta=0.125$ 0.23 for $2 \mathrm{D} \mathrm{XY}$ criticality (21). Furthermore, the specific heat exponent $\alpha=-0.28 \pm 0.04$ is more negative than the $\alpha=-0.13$ to -0.198 predicted for classical, dipolar, and quantum 3D Heisenberg models. Both exponents are consistent with recent Monte-Carlo data on a dipolar 2D bi-layer square lattice finding $\beta=0.18 \pm 0.02$ and $\alpha \simeq-0.4 \pm 0.2$ (22). Combining the Rushbrooke and Widom relations yields the exponent $\delta=(2-\alpha) / \beta-1$, which describes the critical behavior of the (antiferromagnetic) order parameter versus (staggered) field $M \propto H^{\frac{1}{\delta}}$ at the transition. Together with $\eta=2-d(\delta-1) /(\delta+1), \delta$ is super-universal depending only 
on the spatial dimension: $\delta=4.7, \eta=0.03$ for 3D (Ising, XY, and Heisenberg) and $\delta=15$, $\eta=0.25$ for $2 \mathrm{D}$ (Ising and XY/h $h_{4}$ ). The exponent $\delta=14.2$ for $\mathrm{LiErF}_{4}$ is close to the $2 \mathrm{D}$ values. If the anomalous scaling dimension $\eta$ of the spatial correlation function $\left\langle S_{0} S_{r}\right\rangle \propto|r|^{d-2+\eta}$ at the critical point is zero, the critical properties can be derived by straightforward dimensional analysis. Assuming 2D fluctuations in $\mathrm{LiErF}_{4}$ yields $\eta=0.26$, signaling strong fluctuations, consistent with the large reduction in transition temperature compared to the MF prediction.

The Mermin-Wagner theorem excludes long-range order in pure 2D XY models, but even infinitesimal 4-fold $\left(h_{4}\right)$ anisotropy leads to conventional order slightly above the KosterlitzThouless transition. Weak $h_{4}$ anisotropy results in the effective exponents $\eta \simeq 0.35$ and $\beta \simeq$ 0.23 (21), which on increasing $h_{4}$ approach $\eta=0.25$ and $\beta=0.125$ - the Onsager solution for 2D Ising magnets. The Onsager solution also predicts a transition temperature reduced from the MF expectation by a factor of $T_{c} / T_{c}^{\mathrm{mf}}=1 / 2 \log (1+\sqrt{2}) \simeq 0.56$ for a NN square lattice model. This is close to our experimental value of $T_{N} / T_{N}^{\mathrm{mf}}=0.52 \mathrm{in} \mathrm{LiErF}_{4}$, but a theoretical effort is needed to generalize the Onsager solution to dipolar compounds.

It is surprising that we obtain 2D quasi-Ising-like exponents for a system whose two-dimensionality is not apparent from simple inspections of the direct and reciprocal crystal lattices, and where the local symmetry was believed to be XY-like. For our data to be related to these models, two ingredients are needed: (1) reduction of spatial dimensionality from three to two, and (2) reduction of spin space dimensionality from one (XY) to zero (Ising). We leave the origins of these dimensional reductions as a topic for future theoretical efforts, noting here only that quantum fluctuations acting in concert with the tensorial nature of the dipolar interaction could give rise to (2) through the phenomenon of order-by-disorder $(23,24)$. Indeed, an estimate of the $h_{4}$ anisotropy due to order-by-disorder is of the correct order of magnitude to yield $\beta=0.15$ (see SOM for details). The long history of theoretical studies of the 2D dipolar-coupled rotor model was recently revived by advances in microfabricated artificial nanomagnet arrays, which are 
pursued both as model systems for fundamental physics (25) and for ultra-high-density magnetic storage technology $(26,27)$. $\mathrm{LiErF}_{4}$, which we see falls into this universality class, now provides a bulk material with a fully determined Hamiltonian where theoretical predictions can be guided and tested.

Turning to the quantum phase transition we observe an order parameter exponent $\beta_{H}=$ $0.31 \pm 0.02$ consistent with classical 3D scaling, thus confirming the long-standing Hertz result that a QPT in a d-dimensional system (2D XY $/ h_{4}$ in our case) scales as a classical system in $d+1$ dimensions (28). The detailed shape of the phase boundary was determined from susceptibility measurements (Fig. 4E and 4F). Above $2 \mathrm{kOe}, T_{N}(H)$ scales as a power law with exponent $0.34 \pm 0.01$. At $H_{c}, \chi(T)$ exhibits quantum critical scaling, following a power-law exponent $0.70 \pm 0.03$ up to $250 \mathrm{mK}$, above which it crosses over to classical Curie-Weiss behaviour. This behaviour around the QCP is in stark contrast to the MF behaviour observed in $\mathrm{FM} \mathrm{LiHoF}_{4}$. Interestingly, the exponent is close to the 0.75 reported for the heavy Fermion metal $\mathrm{CeCu}_{6-x} \mathrm{Au}_{x}$ near quantum criticality (29).

For $\mathrm{LiHo}_{x} \mathrm{Y}_{1-x} \mathrm{~F}_{4}$, much recent theoretical interest focused on random fields, off-diagonal terms of the dipole interaction, and the emergence of glassiness (30-32). We expect $\mathrm{LiErF}_{4}$ to show dramatic effects of dilution with nonmagnetic ions, or enhancement of off-diagonal terms via substitution of Ho for Er ions. An added benefit of Er is the existence of isotopes with and without nuclear spins, allowing comparative exploration of decoherence and mixing effects (33, 34). Compared to other insulating or itinerant systems, $\mathrm{LiErF}_{4}$ has the advantage of a simple, well-characterized Hamiltonian and of being available in large, high-quality single crystals; it promises insights into the fundamental science of quantum dipolar antiferromagnetism. 


\section{References}

1. J.M. Luttinger and L. Tisza. Theory of Dipole Interaction in Crystals. Phys. Rev., 70:954, 1946.

2. J. Als-Nielsen. Experimental Test of Renormalization Group Theory on the Uniaxial, Dipolar Coupled Ferromagnet LiTbF 4 . Phys. Rev. Lett., 37:1161, 1976.

3. R. Giraud, W. Wernsdorfer, A.M. Tkachuk, D. Mailly, and B. Barbara. Nuclear Spin Driven Quantum Relaxation in $\mathrm{LiY}_{0.998} \mathrm{Ho}_{0.002} \mathrm{~F}_{4}$. Phys. Rev. Lett., 87:057203, 2001.

4. W. Wu, B. Ellman, T.F. Rosenbaum, G. Aeppli, and D.H. Reich. From Classical to Quantum Glass. Phys. Rev. Lett., 67:2076, 1991.

5. P.E. Jönsson, R. Mathieu, W. Wernsdorfer, A.M. Tkachuk, and B. Barbara. Absence of conventional spin-glass transition in the Ising Dipolar System $\mathrm{LiHo}_{x} \mathrm{Y}_{1-x} \mathrm{~F}_{4}$. Phys. Rev. Lett., 98:256403, 2007.

6. J.A. Quilliam, S. Meng, C.G.A. Mugford, and J.B. Kycia. Evidence of spin glass dynamics in dilute $\mathrm{LiHo}_{x} \mathrm{Y}_{1-x} \mathrm{~F}_{4}$. Phys. Rev. Lett., 101:187204, 2008.

7. D. Bitko, T.F. Rosenbaum, and G. Aeppli. Quantum Critical Behavior for a Model Magnet. Phys. Rev. Lett., 77:940, 1996.

8. D.H. Reich, B. Ellman, J. Yang, T.F. Rosenbaum, G. Aeppli, and D.P. Belanger. Dipolar magnets and glasses: Neutron-scattering, dynamical, and calorimetric studies of randomly distributed Ising spins. Phys. Rev. B, 42:4631, 1990.

9. J. Jensen and A.R. Mackintosh. Rare earth magnetism. Clarendon Oxford, 1991. 
10. H.M. Rønnow, J. Jensen, R. Parthasarathy, G. Aeppli, T.F. Rosenbaum, D.F. McMorrow, and C. Kraemer. Magnetic excitations near the quantum phase transition in the Ising ferromagnet $\mathrm{LiHoF}_{4}$. Phys. Rev. B, 75:054426, 2007.

11. J.P. Sattler and J. Nemarich. Electron-Paramagnetic-Resonance Spectra of $\mathrm{Nd}^{3+}, \mathrm{Dy}^{3+}$, $\mathrm{Er}^{3+}$, and $\mathrm{Yb}^{3+}$ in Lithium Yttrium Fluoride. Phys. Rev. B, 4:1, 1971.

12. M.R. Brown, K.G. Roots, and W.A. Shand. Energy levels of $\mathrm{Er}^{3+}$ in $\mathrm{LiYF}_{4}$. Journal of Physics C: Solid State Physics, 2:593, 1969.

13. J. Magariño, J. Tuchendler, P. Beauvillain, and I. Laursen. EPR experiments in $\mathrm{LiTbF}_{4}$, $\mathrm{LiHoF}_{4}$, and $\mathrm{LiErF}_{4}$ at submillimeter frequencies. Phys. Rev. B, 21:18, 1980.

14. P.E. Hansen, T. Johansson, and R. Nevald. Magnetic properties of lithium rare-earth fluorides: Ferromagnetism in $\mathrm{LiErF}_{4}$ and $\mathrm{LiHoF}_{4}$ and crystal-field parameters at the rare-earth and Li sites. Phys. Rev. B, 12:5315, 1975.

15. H.P. Christensen. Spectroscopic analysis of $\mathrm{LiHoF}_{4}$ and $\mathrm{LiErF}_{4}$. Phys. Rev. B, 19:6564, 1979.

16. P. Beauvillain, J.P. Renard, and P.E. Hansen. Low-temperature magnetic susceptibility of $\mathrm{LiErF}_{4}$ : evidence of antiferromagnetic ordering at 0.38K. J. Phys. C, 10:L709, 1977.

17. G. Mennenga, L.J. de Jong, and W.J. Huiskamp. A comparative study of the magnetic ordering specific heats of four $\mathrm{S}=1 / 2$ dipolar magnets: $\mathrm{LiRF}_{4}(\mathrm{R}=\mathrm{Er}, \mathrm{Dy}, \mathrm{Ho}, \mathrm{Tb}) . J M M M$, 44:48, 1984.

18. Sushil.K. Misra and Joshua. Felsteiner. Low-temperature ordered states of lithium rareearth tetrafluorides $\left(\mathrm{LiRF}_{4}\right)$. Phys. Rev. B, 15:4309, 1977. 
19. Guenter. Ahlers, Avinoam. Kornblit, and H.J. Guggenheim. Logarithmic Corrections to the Landau Specific Heat near the Curie Temperature of the Dipolar Ising Ferromagnet LiTbF4. Phys. Rev. Lett., 34:1227, 1975.

20. James. Nikkel and Brett. Ellman. Testing renormalization group theory at the critical dimension in $\mathrm{LiHoF}_{4}$. Phys. Rev. B, 64:214420, 2001.

21. A. Taroni, S.T. Bramwell, and P.C.W. Holdsworth. Universal window for two-dimensional critical exponents. Journal of Physics: Condensed Matter, 20:275233, 2008.

22. L.A.S. Mól and B.V. Costa. Anisotropic Heisenberg model with dipolar interactions: Monte Carlo simulations of the planar-to-paramagnetic phase transition in a bilayer system. Phys. Rev. B, 79:054404, 2009.

23. J. Villain, R. Bidaux, J.P. Carton, and R. Conte. Order as an effect of disorder. J. Phys. (Paris), 41:1263, 1980.

24. Christopher.L. Henley. Selection by Quantum Fluctuations of Dipolar Order in a Diamond Lattice. Phys. Rev. Lett., 73:2788, 1994.

25. R.F. Wang, C. Nisoli, R.S. Freitas, J. Li, W. McConville, B.J. Cooley, M.S. Lund, N. Samarth, C. Leighton, and V.H. Crespi. Artificial spin ice in a geometrically frustrated lattice of nanoscale ferromagnetic islands. Nature, 439:303, 2006.

26. J.I. Martín, J. Nogués, K. Liu, J.L. Vicent, and Ivan.K. Schuller. Ordered magnetic nanostructures: fabrication and properties. J. Mag. Mag. Mat., 256:449, 2003.

27. M.V. Lubarda, S. Li, B. Livshitz, E.E. Fullerton, and V. Lomakin. Antiferromagnetically coupled capped bit patterned media for high-density magnetic recording. Appl. Phys. Lett., 98:012513, 2011. 
28. A.J. Hertz. Quantum critical phenomena. Phys. Rev. B, 14:1165, 1976.

29. A. Schröder, G. Aeppli, E. Bucher, R. Ramazashvili, and P. Coleman. Scaling of Magnetic Fluctuations near a Quantum Phase Transition. Phys. Rev. Lett., 80:5623, 1998.

30. D.M. Silevitch, D. Bitko, J. Brooke, S. Ghosh, G. Aeppli, and T.F. Rosenbaum. A ferromagnet in a continuously tunable random field. Nature, 448:567, 2007.

31. M. Schechter and N. Laflorencie. Quantum Spin Glass and the Dipolar Interaction. Phys. Rev. Lett., 97:137204, 2006.

32. S.M.A. Tabei, M.J.P. Gingras, Y.J. Kao, P. Stasiak, and J.Y. Fortin. Induced Random Fields in the $\mathrm{LiHo}_{x} \mathrm{Y}_{1-x} \mathrm{~F}_{4}$ Quantum Ising Magnet in a Transverse Magnetic Field. Phys. Rev. Lett., 97:237203, 2006.

33. N.V. Prokof'ev and P.C.E Stamp. Theory of the spin bath. Reports on Progress in Physics, 63:669, 2000.

34. H.M. Rønnow, R. Parthasarathy, J. Jensen, G. Aeppli, T.F. Rosenbaum, and D.F. McMorrow. Quantum Phase Transition of a Magnet in a Spin Bath. Science, 308:389, 2005.

We gratefully acknowledge fruitful discussions with J. Mesot, J. Jensen, A. J. Fisher, S. T. Bramwell, and S. Sachdev, support from Swiss National Science Foundation, and MaNEP, and neutron beam access at BENSC, Helmholz-Zentrum Berlin, and SINQ, Paul Scherrer Institut. Work at the University of Chicago was supported by DOE Basic Energy Sciences and the NSF Materials Research Science \& Engineering Center, and that at the London Centre for Nanotechnology by the UK Engineering and Physical Sciences Research Council. 

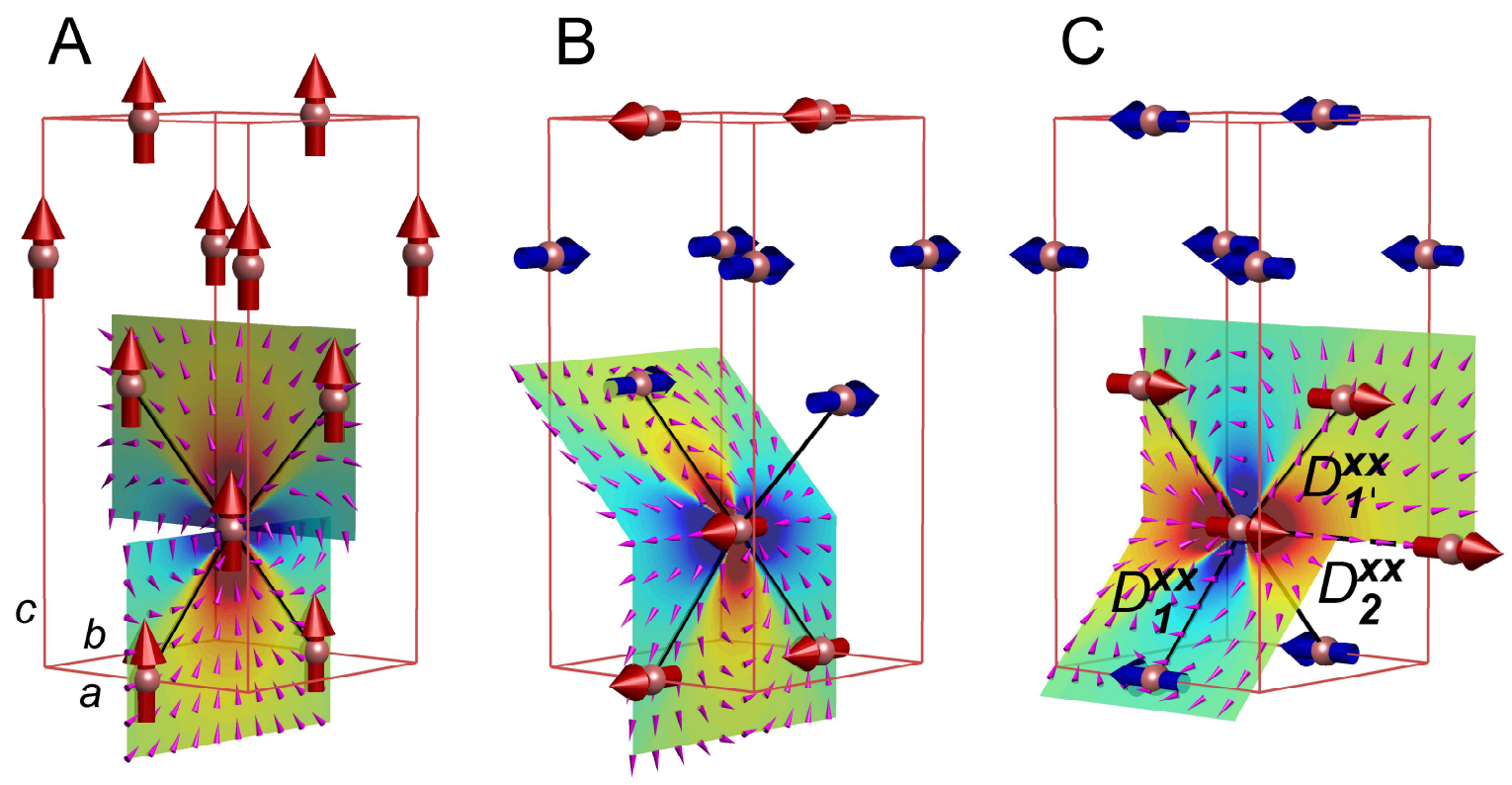

Figure 1: Magnetic structures of $\mathrm{LiREF}_{4}$ : (A) ferromagnetic $c$-axis order in $\mathrm{LiHoF}_{4}$, and bilayered antiferromagnetic (BLAFM) order with moments along (B) $x$ - or (C) $y$-axis in $\mathrm{LiErF}_{4}$. The dipole field from the central moment yields FM (red-scale) and AFM (blue-scale) coupling. Sign and strengths of the coupling depends on the direction of the moments. In the BLAFM structure, nearest and next-nearest couplings are $v D_{1}^{x x}=-5.5(\mathrm{AFM}), v D_{1^{\prime}}^{x x}=2.5(\mathrm{FM})$ and $v D_{2}^{x x}=4.2, v=a^{2} c$. The crystal structure is tetragonal, space group $\mathrm{I} 4_{1} / \mathrm{a}$ with $a=b=$ $5.162 \AA$ and $c=10.70 \AA$. 

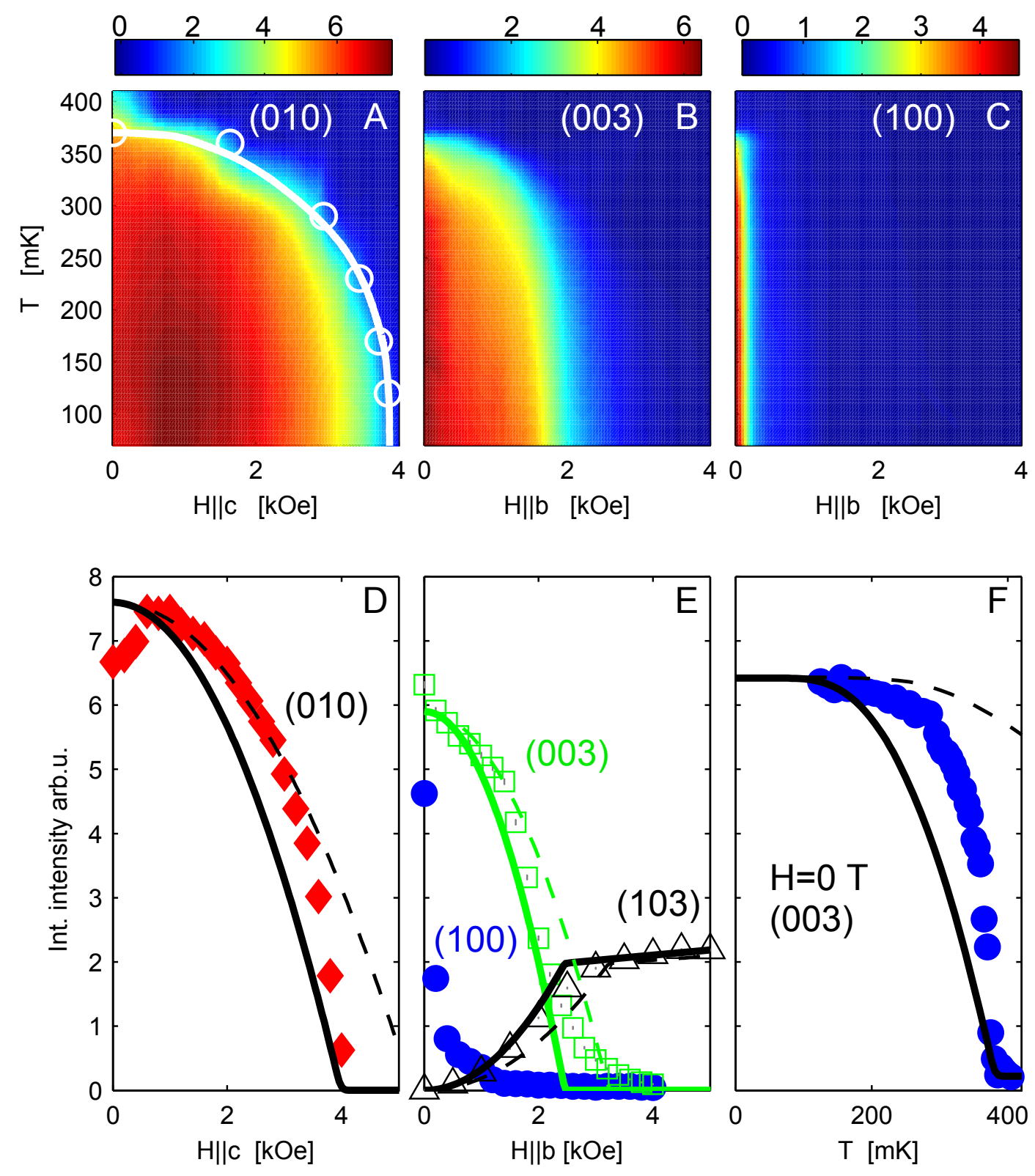

Figure 2: (A-C) Field-temperature phase-diagrams from the intensity of magnetic Bragg peaks: (010) with $H \| c$, (003) and (100) with $H \| b$, respectively. (D,E) Field dependence of peak intensities at $T=100 \mathrm{mK}$ for field along $c$ : (010) and along $b$ : (100), (103), (003), respectively. (F) Temperature dependence of the (003) intensity. In (D-F), dashed lines are mean-field predictions, also shown in solid with temperature and field axes scaled by 0.52 and 0.76 , respectively, to match the measured $T_{N}$ and $H_{c}$. 


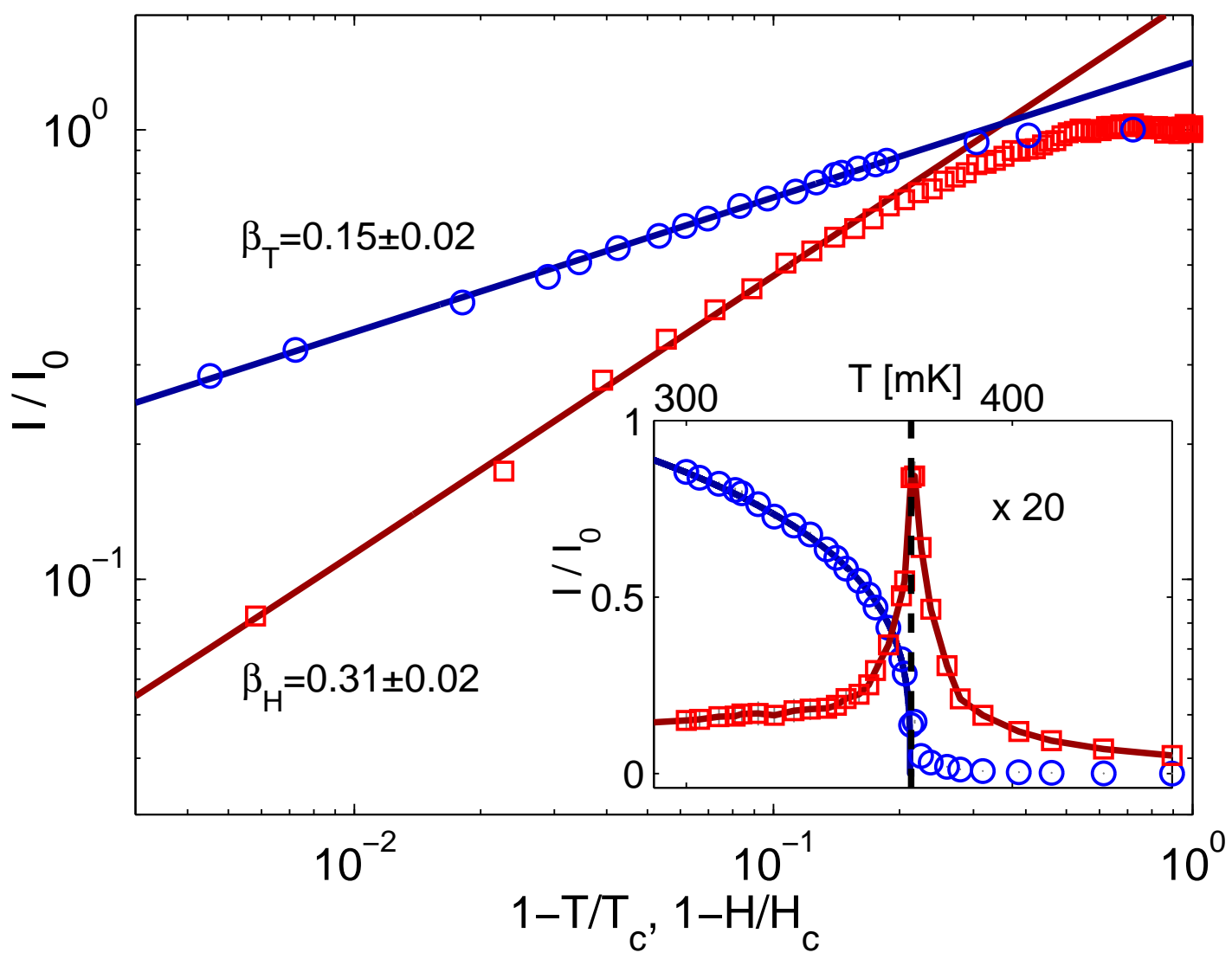

Figure 3: (010) intensity as a function of temperature at $H=0$ (blue circles) and a $c$-axis field at $T=80 \mathrm{mK}$ (red squares). Lines are power law fits. Inset: Intensity of Bragg peak (blue circles) and critical scattering (red squares) extracted by fitting a resolution corrected sum of a delta function and a Lorentzian to crystal rotation scans. 

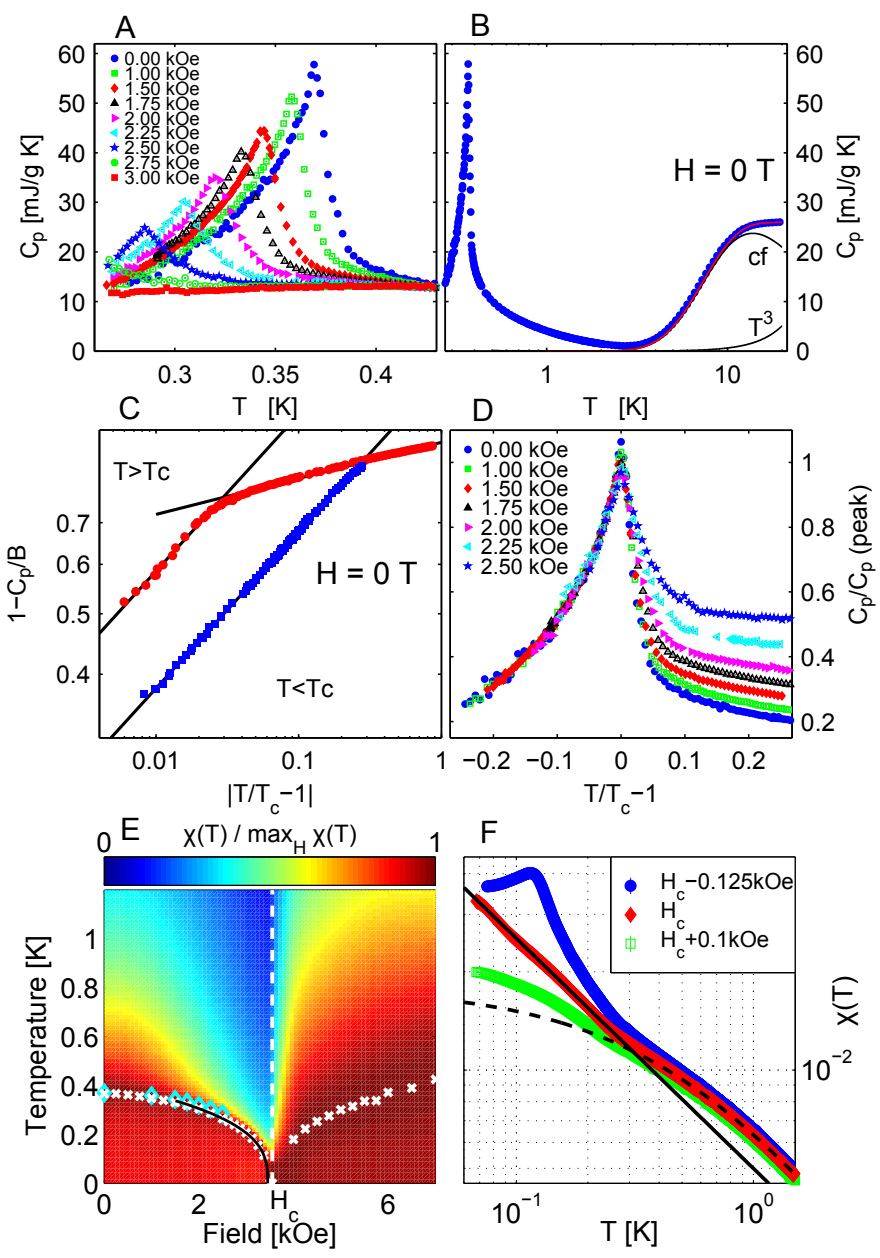

Figure 4: (A) Specific heat versus temperature for several fields along the $c$-axis. (B) Above $2 \mathrm{~K}$, specific heat is described by the sum (red line) of the crystal field Shottky anomaly (cf) and a very weak $T^{3}$ phonon contribution. (C) Power law behavior of specific heat at $H=0 \mathrm{~T}$ determines critical exponents. (D) Curves measured at different field values shifted to the same peak center and normalized by peak height to compare the evolution of peak shape. Below $T_{N}$ the data collapse onto a unique curve. (E) Susceptibility $\chi(T)$ normalized for each field. Peak positions are marked by crosses $(\chi(T))$ and diamonds $\left(C_{p}(T)\right)$. Black line is power law fit to $T_{N}(H)$. (F) $\chi(T)$ just below, at and just above $H_{c}$. At $H_{c}, \chi(T)$ displays quantum critical scaling with exponent $0.70 \pm 0.03$ (solid line), up to a crossover around $250 \mathrm{mK}$, above which regular Curie-Weiss behaviour $C /\left(T-\theta_{C W}\right)$ with $\theta_{C W}=-0.55 \pm 0.01 \mathrm{~K}$ describes the data (dashed line). 